\title{
Percepción de factores modificables que afectan la educación médica en Honduras: Encuesta 2019.
}

\author{
Perception of modifiable factors affecting medical education in Honduras: survey 2019. \\ Jhiamluka Zservando Solano Velásquez ${ }^{1}$ \\ 'Doctor en Medicina y Cirugía, egresado de la Facultad de Ciencias Médicas UNAH, abril, 2018; \\ Clinical Fellow, Queen's Hospital, Barking, Havering and Redbridge University Hospital Trust, Londres; \\ pasante de la Maestría en Educación Médica en University of South Wales, Gales, Reino Unido.
}

\begin{abstract}
RESUMEN. Antecedentes: El sistema de salud en Honduras contribuye de manera importante a la formación médica, facilitando espacios, talento humano y pacientes. Objetivo: Describir la percepción de médicos en formación y docentes sobre factores que pueden afectar la educación médica y la seguridad del paciente, Honduras, 2019. Métodos: Estudio descriptivo transversal con análisis de asociación, en estudiantes de grado/postgrado y docentes, Carrera de Medicina de tres universidades. Se estimó muestra aleatoria. Se realizó encuesta mediante plataforma web disponible durante una semana. Resultados: Se contó con un total de 324 participantes, 205 (63.3\%) estudiantes de grado (7mo y 8avo años), 102 (31.5\%) estudiantes de postgrado y 17 (5.3\%) docentes; $53.4 \%$ (173) pertenecía al género femenino. De los estudiantes, 33.9\% (104) informó sufrir acoso sexual, $36.8 \%$ (113) sufrir sanciones no reglamentadas, $47.9 \%$ (147) realizar procedimientos no enseñados y sin supervisión. El 58.8\% (10) de los docentes consideró que el tiempo estipulado para enseñar no es suficiente en contraste con otras obligaciones docentes. El $42.3 \%$ (130) de los estudiantes y $11.8 \%$ (2) de los docentes presentaron Síndrome de Burnout severo. La sobrecarga laboral se asoció a no adquirir nuevas habilidades en estudiantes de $7 \mathrm{mo}$ año (OR 2.5, IC95\% 1.2-3.7, $p=0.02$ ) y a faltar a clases mandatorias en estudiantes de $7 \mathrm{mo}$ año y residentes en un 75.1\% (OR 3.4, IC95\% 2.82-3.98, p=0.05). Discusión: La sobrecarga laboral, el acoso sexual y laboral, las sanciones no reglamentados y el Síndrome de Burnout son factores que al modificarlos pueden contribuir a una impostergable mejora de la educación médica en Honduras.
\end{abstract}

Palabras claves: Agotamiento psicológico, Educación basada en competencias, Educación médica, Seguridad del paciente.

\section{INTRODUCCIÓN}

La evaluación constante de los programas de formación médica ha demostrado el impacto que la formación médica tiene en los médicos y los pacientes. ${ }^{1}$ Además, nos muestra los retos y posibles soluciones. Las evaluaciones mediante sistemas de retroalimentación a través de percepciones de médicos en formación y docentes son una forma de mejorar los procesos de aprendizaje donde podemos evaluar al estudiante y docente, pero también el ambiente donde se lleva a cabo este proceso. ${ }^{2}$ El docente es una de las variables más significativas, sin embargo, la búsqueda de metodologías complementarias e innovativas es imperativo para todas las academias médicas del mundo. ${ }^{3}$ El modelo por competencias haciendo referencia a conocimientos, habilidades y actitudes para llevar a cabo 0 intervenir en un proceso, resultado de su integración y que han sido adquiridos en el proceso de aprendizaje es importante, ${ }^{4}$ sin embargo, el ambiente donde este se construye debe tener ciertas características para lograr un resultado óptimo.

En Honduras, actualmente existen tres universidades, una pública y dos privadas, que ofrecen formación médica. El modelo

Recibido: 17-10-2019; Aceptado para publicación 16-10-2019

Dirección para correspondencia: Dr. Jhiamluka Solano,

Correo electrónico: jhiamv@gmail.com

Conflictos de interés. El autor declaran no tener conflictos de interés en relación a este artículo.

DOI: https://doi.org/10.5377/rmh.v87i2.11902 actual de formación médica está organizado por departamentos. ${ }^{5}$ En 1971 se realizaron reformas al plan de estudios original a fin de basarlo en objetivos de aprendizaje, similar al modelo Miller de 1990 fundamentado en el enfoque evaluativo de las competencias propias de un médico clínico. ${ }^{6,7}$ En 1975 se crearon las primeras residencias médicas. ${ }^{8}$ En la actualidad, ninguna de las universidades que ofrecen formación médica en Honduras cuenta con un sistema de retroalimentación, por parte de los médicos en formación y de los docentes, que evalúe componentes no académicos importantes en los procesos de formación en el ambiente clínico, como la satisfacción general, supervisión clínica, experiencia clínica, acoso laboral, sanciones, abuso sexual, apoyo emocional, Síndrome de Burnout y la seguridad del paciente. Fundamentado en lo anterior, este estudio se realizó con el objetivo de describir la percepción de médicos en formación y de docentes de Honduras sobre factores modificables que pueden afectar la educación médica y la seguridad del paciente, con la finalidad de contribuir a desarrollar estrategias que permitan mejorar la educación médica en el país por parte de las diferentes instituciones involucradas en la formación, regulación y supervisión de los médicos en formación.

\section{METODOLOGÍA}

Se realizó un estudio descriptivo transversal con análisis de asociación sobre la percepción de médicos en formación y 
de docentes de Honduras sobre factores modificables que pueden afectar la educación médica y la seguridad del paciente. El estudio incluyó médicos en formación de grado y postgrado de la Carrera de Medicina y docentes de la Universidad Nacional Autónoma de Honduras, Universidad Católica de Honduras y la Universidad Tecnológica Centroamericana. El estudio se realizó en el mes de agosto del año 2019. Se utilizó una encuesta Internet a través de la plataforma web Qualtrics (https://www.qualtrics.com/) que permite el análisis de datos. En este estudio no se evaluaron aspectos académicos propios del plan académico, ni los contenidos o la metodología de enseñanza; tampoco se trata de un estudio para evaluar los conocimientos, actitudes y prácticas sobre un tema en particular o pensum académico de los participantes.

Para los médicos en formación, se estimó una muestra estratificada por nivel de formación utilizando estimaciones del nivel de confianza y margen de error. Se contaba con 813 estudiantes de 7mo año (Internado Rotatorio), con una muestra de 262 (Nivel de Confianza 95\%, Margen de Error 5\%), 1,653 estudiantes de 8 avo año (Servicio Médico Social Obligatorio) con una muestra de 312 (Nivel de Confianza 95\%, Margen de Error 5\%), 388 médicos residentes con una muestra de 194 (Nivel de Confianza 95\%, Margen de Error 9.5\%). Para los docentes, se contaba con 61 docentes contratados y asignados al internado rotatorio y residencia médica a nivel nacional, por lo que se estimó incluirlos en su totalidad.

El enlace de acceso a la encuesta se distribuyó a través de grupos de redes sociales cerrados (WhatsApp) de cada nivel académico a nivel nacional. Para los médicos en formación, el instrumento constaba de 9 secciones con preguntas tipo selección única, múltiple y abiertas, generales y específicas en las diferentes secciones, de acuerdo con el tipo de participante. Se registró la siguiente información: 1) Datos Generales, 2) Satisfacción General, 3) Supervisión Clínica, 4) Seguridad del Paciente, 5) Experiencia Clínica, 6) Acoso Laboral, sanciones y abuso sexual, 7) Apoyo emocional, 8) Síndrome de Burnout, mediante cuestionario Maslach Burnout Inventory (MBI), ${ }^{9}$ y 9) Recomendaciones para mejorar el instrumento. Cuando se les solicitó calificar la calidad de enseñanza y entrenamiento, las opciones de calificación utilizadas fueron $25 \%, 50 \%, 75 \%$ y $100 \%$. En el instrumento de los docentes se registró la siguiente información: 1) Datos generales, 2) Satisfacción general, 3) Labor docente, 4) Apoyo emocional, 5) Acoso y abuso laboral/ sexual, 6) Burnout mediante cuestionario Maslach Burnout Inventory $(\mathrm{MBI})^{9}$ 7) Seguridad del paciente y 8) Recomendaciones desde la percepción del docente. Se hizo una prueba piloto con 100 participantes de los diferentes niveles académicos y se tomaron recomendaciones para corrección y modificación del instrumento.

Se realizó análisis estadístico mediante la plataforma web Qualtrics utilizando un software que facilita el análisis para actuar sobre puntos de vista, incluyendo análisis descriptivo bivariado y aplicación de medidas de tendencia central con pro- medios de frecuencias y análisis del índice de Burnout. Se estimó OR e IC95\% incluyendo los factores sobrecarga laboral, la inasistencia a clases y la adquisición de nuevas habilidades. Se consideró significancia estadística con un valor de $p<0.05$.

Se contó con el apoyo institucional del Colegio Médico de Honduras y se siguieron las buenas prácticas en investigación en salud. El protocolo fue validado mediante revisión por estudiantes coordinadores nacionales de estudiantes de 7 mo y 8 avo años y médicos especialistas docentes adscritos al Colegio Médico de Honduras. El estudio no fue sometido a ningún comité de ética en investigación. Se realizó la anonimización completa de los datos trazables de los participantes siguiendo los lineamientos éticos recomendados por la plataforma web (https:// www.qualtrics.com/blog/ethical-issues-for-online-surveys/). Al inicio de la encuesta se brindó información invitando a participar, explicando el objetivo del estudio y el manejo anónimo de la información personal de los participantes. Fundamentado en esta información, el participante tomaba la decisión de participar o no al iniciar el llenado de la encuesta.

\section{RESULTADOS}

Se contó con 324 participantes, en relación con el sexo, el $53.4 \%$ (173) pertenecía al sexo femenino. El grupo de edad más frecuente en los diferentes grupos participantes fue 24-27 años en estudiantes de $7 \mathrm{mo}$ año (61.9\%) y de 8avo año (63.0\%) y 28-31 años en médicos residentes (68.6\%). Entre los médicos residentes, $87.3 \%$ (88) se registraron primer a tercer año. En el Cuadro 1, se presenta el número de participantes según grado académico, especialidad, número de departamentos del país y docentes que participaron en el estudio. Se contó con participantes de 17 Departamentos del país en donde se realiza el Servicio Social y 7 de 9 establecimientos de salud donde se realiza el internado rotatorio. Se contó con participación de docentes de los tres centros universitarios. Los participantes del $7 \mathrm{mo}$ año se encontraban rotando por Medicina Interna (20.4\%), Cirugía General (34.0\%), Pediatría (29.1\%), Ginecología y Obstetricia (16.5\%). El $70 \%$ de los estudiantes de 8avo año pertenecían al Primer Nivel de Atención. En cuanto a la ubicación geográfica, la participación general procedía del departamento de Francisco Morazán (62.0\%) seguido del Departamento de Cortés (12.0\%).

Con relación a la satisfacción general, el 30.3\% (93) informó desconocer el reglamento que regula el nivel académico en el que se encontraba. La formación en su desarrollo personal y profesional sobre aspectos éticos fue considerada inaceptable $(12.9 \%)$, aceptable $(42.7 \%)$, buena $(30.3 \%$ ) y excelente (14.1\%). La carga laboral fue calificada como muy pesada por los estudiantes de $7 \mathrm{mo}$ año (58.1\%), estudiantes de 8avo año $(16.0 \%)$ y médicos residentes $(50.0 \%)$. La sobrecarga laboral (pesada o muy pesada) se asoció a la información registrada de no adquirir nuevas habilidades por estudiantes de $7 \mathrm{mo}$ año (OR 2.5, IC95\% 1.2-3.7, $p=0.02$ ), estudiantes de 8avo año (OR 3.3, 
Cuadro 1. Número de participantes según grado académico, especialidad, número de departamentos del país y docentes que participaron en el estudio, Formación Médica, Honduras, 2019, N= 324.

\begin{tabular}{|c|c|c|}
\hline \multirow[t]{2}{*}{ Nivel Académico } & \multicolumn{2}{|c|}{$\begin{array}{l}\text { NÚMERO DE MÉDICOS } \\
\text { RESIDENTES }\end{array}$} \\
\hline & $\begin{array}{c}\text { Postgrado } \\
\text { Clínico } \\
\text { N (\%) }\end{array}$ & $\begin{array}{c}\text { Participantes } \\
\text { en el Estudio } \\
\mathbf{N}(\%)\end{array}$ \\
\hline Residencia Médica & $388(100.0)$ & $102(26.3)$ \\
\hline Anatomía Patológica & $3(0.8)$ & $1(1.0)$ \\
\hline Anestesiología, Reanimación y Dolor & $31(8.0)$ & $1(1.0)$ \\
\hline Cirugía General & $31(8.0)$ & $16(15.7)$ \\
\hline Cirugía Plástica y Reconstructiva & $2(0.5)$ & $2(2.0)$ \\
\hline Cuidados Intensivos Pediátricos & $4(1.03)$ & $3(2.9)$ \\
\hline Dermatología & $5(1.3)$ & $2(2.0)$ \\
\hline Ginecología y Obstetricia & $70(18.0)$ & $13(12.8)$ \\
\hline Medicina de Rehabilitación & $12(3.1)$ & $10(9.8)$ \\
\hline Medicina Legal y Forense & $5(1.3)$ & $0(0.0)$ \\
\hline Medicina Interna & $63(16.2)$ & $6(5.9)$ \\
\hline Neurocirugía & $6(1.6)$ & $2(2.0)$ \\
\hline Neurología & $10(2.6)$ & $6(5.9)$ \\
\hline Oftalmología & $5(1.3)$ & $2(2.09$ \\
\hline Oncología Quirúrgica & $7(1.8)$ & $3(3.0)$ \\
\hline Ortopedia y Traumatología & $17(4.4)$ & $5(5.0)$ \\
\hline Otorrinolaringología & $10(2.6)$ & $8(7.8)$ \\
\hline Pediatría & $76(19.6)$ & $15(14.7)$ \\
\hline Psiquiatría & $11(2.8)$ & $3(3.0)$ \\
\hline Radiología e Imágenes Médicas & $20(5.2)$ & $4(4.0)$ \\
\hline \multirow[t]{2}{*}{ Otra } & $0(0.0)$ & $0(0.0)$ \\
\hline & $\begin{array}{l}\text { Nivel nacional } \\
\quad \mathbf{N}(\%)\end{array}$ & $\begin{array}{l}\text { Participantes } \\
\quad \text { N (\%) }\end{array}$ \\
\hline Estudiantes 8avo Año & $1,653(100.0)$ & $100(6.5)$ \\
\hline $\begin{array}{l}\text { Departamentos de Honduras donde } \\
\text { se realiza el Servicio Social }\end{array}$ & $18(100.0)$ & $17(94.4)$ \\
\hline Estudiantes 7mo Año & $813(100.0)$ & $105(13.0)$ \\
\hline $\begin{array}{l}\text { Departamentos de Honduras donde } \\
\text { se realiza el Internado Rotatorio }\end{array}$ & $9(100.0)$ & $7(77.8)$ \\
\hline Docentes* & $61(100.0)$ & $17(27.9)$ \\
\hline
\end{tabular}

* Estimado a nivel nacional dedicado entre las 3 universidades designado a Internado Rotatorio y Residencia Médica.

IC95\% 2.5-4.2, $p=0.01$ ) y médicos residentes (OR 3.7, IC95\% $2.5-4.8, p=0.0001$ ). Los participantes informaron retirarse 0 ingresar fuera de horarios durante la semana: estudiantes de $7 \mathrm{mo}$ año, 7 días (58.1\%); estudiantes 8avo año, 3-5 días (45\%) y médicos residentes, 7 días (51.0\%). Las razones más comunes fueron la carga laboral muy pesada según estudiantes de $7 \mathrm{mo}$ año (65.6\%), estudiantes de 8 avo año $(24.4 \%)$ y médicos residentes $(71.2 \%)$; sanción de un superior según estudiantes de 7 mo año (50.8\%), estudiantes de 8avo año (56.5\%) y médicos residentes $(69.2 \%)$.
Con respecto a la supervisión clínica, $81.8 \%$ (252) refirió ser supervisado por un médico graduado o de mayor grado académico, con menor frecuencia referido por estudiantes de 8avo año $(57.0 \%)$. Se registró que la supervisión se da en menos del $50 \%$ del tiempo durante el rol clínico, referido por estudiantes de $7 m o$ año (59.1\%), estudiantes de 8avo año $(90 \%)$, así mismo, en $40 \%$ del tiempo sin ninguna supervisión y los médicos residentes (57.9\%). La retroalimentación fue recibida en menos de la mitad del tiempo supervisado (estudiantes de $7 \mathrm{mo}$ año $77.1 \%$, estudiantes de 8 avo año $90 \%$, médicos residentes $63.7 \%$ ). En el Cuadro 2 se presentan las calificaciones otorgadas sobre la calidad de la enseñanza y entrenamiento de parte del médico de grado superior.

En cuanto a la experiencia clínica, los participantes médicos en formación reportaron que el $81.6 \%$ (251) de los establecimientos de salud en donde estaban asignados no cuenta con suficiente médico asignado en relación con la carga laboral. El $86.9 \%$ (267) considera que la carga laboral no permite la adecuada enseñanza de parte de médicos más experimentados. El $59.9 \%$ (184) indicó que debe faltar a clases para cubrir trabajo clínico a falta de recurso humano en la unidad de salud. Se asoció la sobrecarga laboral y faltar a clases en un $75.1 \%$ (OR 3.4 , IC95\% 2.82-3.98, $p=0.05$ ). En cuanto a las responsabilidades según grado académico, el $66.5 \%$ (204) refirió se le ha dado la responsabilidad acorde al nivel de formación en que se encuentra. Sin embargo, $63.2 \%$ (194) registró que se le ha solicitado realizar tareas que van más allá de sus competencias o responsabilidades según su nivel de formación.

En el Cuadro 3, se describen las características del acoso sexual por su frecuencia, tipo de acoso y perpetrador. El 33.9\%

Cuadro 2. Distribución de la calificación de la calidad de la enseñanza y entrenamiento por parte del médico de grado superior otorgada por estudiantes de 7 mo Año de la Carrera de Medicina y estudiantes de los postgrados clínicos, Formación Médica, Honduras, 2019, n= 207.

\begin{tabular}{|c|c|c|}
\hline \multirow{2}{*}{$\begin{array}{c}\text { Calificacion de la calidad de la } \\
\text { enseñanza y entrenamiento por } \\
\text { parte del médico de grado su- } \\
\text { perior }\end{array}$} & \multicolumn{2}{|c|}{ Participante que califico } \\
\hline & $\begin{array}{l}\text { Estudiante de } \\
7 \text { mo año } \\
\mathrm{N}(\%)\end{array}$ & $\begin{array}{l}\text { Médico resi- } \\
\text { dente } \\
\mathrm{N}(\%)\end{array}$ \\
\hline \multicolumn{3}{|l|}{ Visita Hospitalaria } \\
\hline $25 \%$ & $39(37.5)$ & $23(22.8)$ \\
\hline $50 \%$ & $30(28.9)$ & $29(28.7)$ \\
\hline $75 \%$ & $25(24.0)$ & $36(36.6)$ \\
\hline $100 \%$ & $10(9.6)$ & $13(12.9)$ \\
\hline \multicolumn{3}{|l|}{ Atención en Consulta Externa* } \\
\hline $25 \%$ & $32(30.8)$ & $25(16.8)$ \\
\hline $50 \%$ & 33 (31.7) & $29(28.7)$ \\
\hline $75 \%$ & $31(29.8)$ & $35(34.7)$ \\
\hline $100 \%$ & $8(7.7)$ & $12(11.9)$ \\
\hline \multicolumn{3}{|l|}{ Tiempo de quirófano** } \\
\hline $25 \%$ & $37(35.6)$ & $24(23.8)$ \\
\hline $50 \%$ & $25(24.0)$ & $23(22.8)$ \\
\hline $75 \%$ & $28(27.0)$ & $25(24.8)$ \\
\hline $100 \%$ & $14(13.5)$ & $18(17.8)$ \\
\hline
\end{tabular}

*No aplica en $8.0 \%$ (8) de médicos residentes; ** No aplica en 10.9\% (11) de médicos residentes. 
Cuadro 3. Características del acoso sexual descritos por estudiantes de $7 \mathrm{mo}$ y 8 avo años de la Carrera de Medicina y Médicos Residentes, Formación Médica, Honduras, 2019, $n=104$.

\begin{tabular}{|c|c|c|c|}
\hline \multirow{2}{*}{$\begin{array}{c}\text { Caracteristicas del acoso } \\
\text { sexual }\end{array}$} & \multicolumn{3}{|c|}{ Participante que refirio acoso sexual } \\
\hline & $\begin{array}{c}\text { Estudiante de } \\
7 \text { mo año } \\
N(\%)\end{array}$ & $\begin{array}{c}\text { Estudiante } \\
\text { de 8avo año } \\
\mathrm{N}(\%)\end{array}$ & $\begin{array}{c}\text { Médico } \\
\text { residente } \\
\mathbf{N}(\%)\end{array}$ \\
\hline $\begin{array}{l}\text { Frecuencia } \\
\text { Nunca } \\
\text { Rara vez } \\
\text { Frecuentemente }\end{array}$ & $\begin{array}{l}54(51.4) \\
30(28.6) \\
21(20.0)\end{array}$ & $\begin{array}{l}74(74.0) \\
20(20.0) \\
6(6.0)\end{array}$ & $\begin{array}{l}75(73.5) \\
21(20.6) \\
6(5.9)\end{array}$ \\
\hline $\begin{array}{l}\text { Tipo } \\
\text { Actos de violencia física } \\
\text { Verbal a }^{\text {a }} \\
\text { Gestos de naturaleza sexual }^{b} \\
\text { Envío de mensajes }^{c} \\
\text { Difamación sobre su vida sexual }\end{array}$ & $\begin{array}{l}9(17.7) \\
33(64.7) \\
4(7.9) \\
3(5.9) \\
2(4.0)\end{array}$ & $\begin{array}{l}2(7.7) \\
18(69.2) \\
2(7.7) \\
1(3.9) \\
3(11.5)\end{array}$ & $\begin{array}{l}4(9.3) \\
22(51.2) \\
9(21.0) \\
4(9.3) \\
4(9.3)\end{array}$ \\
\hline $\begin{array}{l}\text { Perpetrador } \\
\text { Médicos Especialistas } \\
\text { Médicos Residentes } \\
\text { Personal asistencial no médico } \\
\text { Pacientes }\end{array}$ & $\begin{array}{l}32(28.6) \\
20(17.9) \\
17(15.2) \\
0(0.0)\end{array}$ & $\begin{array}{l}8(15.7) \\
0(0.0) \\
13(25.5) \\
11(21.6)\end{array}$ & $\begin{array}{l}14(26.5) \\
10(18.9) \\
9(17.0) \\
0(0.0)\end{array}$ \\
\hline $\begin{array}{l}\text { Rotación de Internado } \mathrm{N}=1 \mathbf{5}^{\mathrm{d}} \\
\text { Cirugía } \\
\text { Pediatría } \\
\text { Medicina Interna } \\
\text { Ginecología y Obstetricia }\end{array}$ & $\begin{array}{l}40(38.1) \\
29(27.6) \\
19(18.1) \\
17(16.2)\end{array}$ & & \\
\hline
\end{tabular}

Comentarios, piropos, supuesto cumplidos, chantaje, apelaciones al aspecto físico o vida privada b Silbidos, picos, señas, miradas

c Contenido (fotos de personas desnudas) y palabras inapropiadas.

${ }^{d} A$ coso sexual según rotación de internado rotatorio en la población de estudiantes de 7 mo año.

(104) de los médicos en formación expresaron haber experimentado acoso sexual de parte de un superior, compañero(a) o personal no asistencial. Las mujeres informaron experimentar acoso sexual en $21.2 \%$ (65) y los hombres en $13.0 \%$ (40). El $26.1 \%$ (80) consideró que sufrir acoso sexual influyó en su desempeño laboral y su formación profesional. El uso de lenguaje sexual en el ambiente laboral fue referido por $31.0 \%$ (95) de los médicos en formación. Las presiones para aceptar citas a tomar copas o cenar con propósitos sexuales se registró en $33.7 \%$ (32) de los casos de acoso sexual.

El 36.8\% (113) de los médicos en formación informó haber sido sancionado de maneras no contempladas por el reglamento respectivo, así como se detalla en el Cuadro 4. Los médicos residentes informaron sanciones no contemplados en la normativa, así: en el primer año 16.6\% (17), segundo año 15.7\% (16), tercer año $12.7 \%$ (13) y cuarto año $2.0 \%$ (2). En relación con la especialidad, los casos se distribuyeron de la siguiente manera: 14.7\% (15) Cirugía General, 8.8\% (9) Ginecología y Obstetricia, 7.8\% (8) Pediatría, 4.9\% (5) Neurología, 3.9\% (4) Ortopedia y Traumatología, 2.9\% (3) Medicina Interna y otras especialidades $5.9 \%$ (6); un total de 13 especialidades y subespecialidades.

Con respecto al apoyo emocional y respeto, $64.2 \%$ (197) informó que fue maltratado de manera verbal por parte de un compañero o superior; por ejemplo, insultos y tono de voz elevado. El 10.4\% (32) fue maltratado de manera física por parte de un compañero o superior; $70.0 \%$ (215) fue maltratado de manera psicológica por parte de un compañero o superior; por ejemplo, demeritar sus competencias o capacidades. El 59.3\% (182) sintió que violentan su integridad personal y profesional de parte de un superior o compañero. El $65.5 \%$ (201) refirió que no se respeta el tiempo establecido para suplir las necesidades básicas humanas (comer, asearse, dormir, usar el baño), con una mayor frecuencia en el $7 \mathrm{mo}$ año $(89.5 \%)$. El $65.8 \%$ (202) de los médicos en formación informó haberse sentido afectado por la muerte o caso de algún paciente y el 2.9\% (9) recibió algún tipo de acompañamiento psicológico. El 13.4\% (41) refirió que sus unidades cuentan con un equipo especializado para dar apoyo de consejería psicológica al personal de la unidad. El 45.9\% (141) informó haber considerado la necesidad de cambiarse de carrera debido al impacto emocional personal y profesional que ha vivido durante su carrera. El $97.7 \%$ (300) consideró importante el acompañamiento psicológico o psiquiátrico.

En cuanto al Síndrome de Burnout severo, se registraron $131(40.4 \%$ ) casos (Ver Cuadro 5). Entre estudiantes de $7 \mathrm{mo}$ año se registró mayor frecuencia en mujeres $67.8 \%$ (42). Las sanciones no reglamentadas estuvieron presentes en $47.2 \%$ (34) de los casos y la carga laboral muy pesada $73.6 \%$ (53) y pesada 26.4 (19). Entre estudiantes de 8 avo año hubo mayor frecuencia en mujeres 10 (71.4\%). Las sanciones no reglamentadas estuvieron presentes en $21.4 \%$ (3) de los casos y la carga laboral moderada en $57.1 \%(8)$ de los casos. Entre los médicos residentes se registró una frecuencia mayor en mujeres $(54.5 \%)$ que en hombres (45.4\%). El 40.9\% (18) de los médicos residentes con Síndrome de Burnout recibió sanciones no reglamentadas y $90.9 \%$ (40) sufría sobrecarga laboral.

Con relación a la seguridad del paciente, el $55.7 \%$ (171) registró que sus unidades no poseen protocolos establecidos para el manejo de pacientes. La entrega de evolución de pacientes en el cambio de guardia se lleva a cabo en $7 \mathrm{mo}$ año en un $68.6 \%$, 8avo año $46.0 \%$ y residencia médica $89.2 \%$. El $25.4 \%$ (78) refirió que se vela por el principio ético de confidencialidad al realizarla en un lugar privado. El 30.9\% (95) registró que existe divulgación de información de pacientes mediante redes sociales. Se registró seguimiento de los incidentes en un $53.7 \%$ (165), con menor frecuencia en el 7 mo año (37.1\%). La reunión de personal para discutir el evento fue registrada como el mecanismo más utilizado 44.2\% (73). El 47.9\% (147) informó haber tenido que realizar procedimientos que no le han sido enseñados y sin supervisión, con mayor frecuencia en el $7 \mathrm{mo}$ año $(61.9 \%)$.

En relación con los docentes el $41.2 \%$ (7) se refirió a la carga laboral como muy pesada, salir después de la hora estipulada en 5 de 7 días de la semana y carecer de guías y protocolos 
Cuadro 4. Sanciones no reglamentadas descritos por estudiantes de 7 mo y 8 avo años de la Carrera de Medicina y Médicos Residentes, Formación Médica, Honduras, $2019, n=307$.

\begin{tabular}{|c|c|c|c|}
\hline TIPO DE SANCIÓN & $\begin{array}{l}\text { PARTICIPANTE } \\
\text { ESTUDIANTE 7MO AÑO } \\
\mathrm{N}=45 \\
\mathrm{~N}(\%)\end{array}$ & $\begin{array}{c}\text { ESTUDIANTE 8AVO } \\
\text { AÑO } \\
\mathrm{N}=17 \\
\mathrm{~N}(\%)\end{array}$ & $\begin{array}{c}\text { MEDICOS } \\
\text { RESIDENTES } \\
\mathrm{N}=51 \\
\mathrm{~N}(\%) \\
\end{array}$ \\
\hline Repetir ingreso sin fundamentos seguido de gesto & $11(24.4)$ & $5(29.4)$ & $28(55.0)$ \\
\hline Retirarse de la unidad después del horario establecido & $32(71.1)$ & $7(41.2)$ & $43(84.3)$ \\
\hline Evitar que se vaya a su casa por uno o más días & $4(8.9)$ & $0(0.0)$ & $22(43.1)$ \\
\hline Realizar tareas que no son parte de sus obligaciones según grado académico & $23(51.1)$ & $5(29.4)$ & $23(45.1)$ \\
\hline Repetir turno después del turno establecido & $7(15.6)$ & $1(5.9)$ & $18(35.3)$ \\
\hline Entrada en horarios no estipulados & $33(73.3)$ & $11(64.7)$ & $24(47.1)$ \\
\hline $\begin{array}{l}\text { Presión para realizar celebraciones de parte de nuevos Médicos Residentes a } \\
\text { Médicos Residentes salientes }\end{array}$ & $5(11.1)$ & $0(0.0)$ & $16(31.3)$ \\
\hline $\begin{array}{l}\text { sanciones Monetarios (Ejemplo: comprarle comida u otras cosas a superiores con } \\
\text { su propio dinero de manera obligatoria) }\end{array}$ & $5(11.1)$ & $1(5.9)$ & $10(19.6)$ \\
\hline Decirse a sí mismo que eran incompetentes con uso lenguaje soez & $23(51.1)$ & $3(17.6)$ & $19(37.3)$ \\
\hline Bañar pacientes o ser camilleros & $28(62.2)$ & $2(11.8)$ & $7(13.7)$ \\
\hline Esconder pacientes para que especialista no lo vean por temor a regaños & $4(8.9)$ & $0(0.0)$ & $7(13.7)$ \\
\hline Utilizar propios recursos para manejo de pacientes (vehículo y dinero) & $19(42.2)$ & $2(11.8)$ & $19(37.3)$ \\
\hline Hacer ingresos que no le corresponden & $17(37.7)$ & $3(17.6)$ & $27(53.0)$ \\
\hline Perder derecho a dormir & $26(57.8)$ & $3(17.6)$ & $23(45.1)$ \\
\hline Otros & $2(4.4)$ & $1(5.9)$ & $4(7.8)$ \\
\hline
\end{tabular}

en la unidad de salud. El 82.4\% (14) considero que no había suficientes médicos asignados a la unidad de salud. El $64.7 \%$ (11) consideró que la carga laboral no le permite suficiente tiempo para enseñar y que es insuficiente el tiempo estipulado para enseñar en contraste con otras obligaciones. El 23.5\% (4) consideró que la segunda causa de inasistencias a clases es la percepción que los médicos en formación son indispensables para el funcionamiento de un servicio, siendo la sobrecarga laboral la principal razón $76.5 \%$ (13). Entre docentes, se registró $11.8 \%$ (2) de casos de acoso sexual de tipo verbal. Se encontró que el $11.8 \%$ (2) de los docentes sufrían de Síndrome de Burnout severo.

\section{DISCUSIÓN}

En este estudio se ha identificado que los médicos en formación y docentes tienen una percepción, conocimiento 0 comprensión sobre factores que pueden afectar la educación médica y la seguridad del paciente en Honduras que debe llamar a la reflexión y a la acción inmediata de los diferentes actores académicos, gubernamentales y sociales involucrados. Las percepciones registradas describen de forma negativa el entorno de relaciones interpersonales y de infraestructura física en el cual se están formando los futuros médicos que atenderán el sistema sanitario, la salud y la formación de talento humano en salud en el país. El actual modelo de formación médica por competencias basado en el triángulo de Miller, ${ }^{7}$ ha demostrado no ser aplicable en Honduras debido a la influencia del sistema y del individuo. Dichos factores son fundamentales en la adquisición y desarrollo de competencias ya que el triángulo de Miller se considera algo rígido porque no toma en consideración lo anteriormente mencionado, por lo cual, es necesario fortalecer el aprendizaje y la reestructuración del rol del sistema de salud en la formación médica. ${ }^{8}$

La educación médica es un proceso para toda la vida que va adoptando una dinámica de desarrollo, revisión y retroalimentación durante la experiencia de educación de pregrado, posgrado y educación médica continua. ${ }^{10}$ En este estudio se ha presentado la evidencia que los médicos en formación carecen de un ambiente académico y su formación se basa mayormente en la exposición clínica o ensayo- error. Este ambiente se encuentra en constante violación de los derechos laborales internacionales establecidos por la OIT y el código del trabajo en Honduras. ${ }^{11}$ Esto se debe a que no se respeta el rol del médico en formación dentro del sistema de salud o no se ha definido.

La sobrecarga laboral, el acoso sexual y la falta de un ambiente académico de respeto registrada por los participantes alcanzan una frecuencia alta en los diferentes niveles de formación (Cuadros 3 y 4). Estos aspectos tienen un impacto negativo en la formación médica, ${ }^{12}$ siendo estos los responsables del desarrollo personal y profesional sobre aspectos éticos y la adquisición de nuevas habilidades. La asistencia a clases mandatorias se ve interrumpida especialmente por la sobrecarga laboral, resaltando que las universidades privadas cuentan con 
Cuadro 5. Casos severos de Síndrome de Burnout de acuerdo con el nivel académico, Formación Médica, Honduras, 2019, $n=324$.

\begin{tabular}{lc}
\hline Nivel académico & $\begin{array}{c}\text { Sindrome de Burnout } \\
\text { severo } \\
\mathrm{N}(\%)\end{array}$ \\
\hline Residencia N= 102 & $44(43.1 \%)$ \\
Pediatría & $9(20.4)$ \\
Cirugía General & $7(15.9)$ \\
Ginecología y Obstetricia & $5(11.4)$ \\
Medicina de Rehabilitación & $4(9.1)$ \\
Neurología & $3(6.8)$ \\
Medicina Interna & $3(6.8)$ \\
Otorrinolaringología & $2(4.6)$ \\
Oftalmología & $2(4.6)$ \\
Ortopedia y Traumatología & $2(4.6)$ \\
Cirugía Plástica y Reconstructiva & $2(4.6)$ \\
Radiología & $1(2.3)$ \\
Psiquiatría & $1(2.3)$ \\
Dermatología & $1(2.3)$ \\
Cuidados Intensivos Pediátricos & $1(2.3)$ \\
Anestesiología, Reanimación y Dolor & $1(2.3)$ \\
Estudiantes 8avo Año, N= 100 & $14(14.0 \%)$ \\
Estudiantes 7mo Año, N= 105 & $71(67.6 \%)$ \\
Cirugía General & $25(35.2)$ \\
Pediatría & $21(29.6)$ \\
Medicina Interna & $15(21.1)$ \\
Ginecología y Obstetricia & $10(14.1)$ \\
\hline Docentes N= 17 & $2(11.8)$ \\
\hline
\end{tabular}

docentes dedicados para impartir clases y la UNAH depende de la modalidad de voluntariado a nivel nacional con excepción del Hospital Escuela y Hospital Mario C. Rivas. Los médicos en formación deben retirarse fuera del horario laboral estipulado constantemente debido a la sobrecarga laboral y sanciones no reglamentadas, hecho injustificado e ilegal.

Adicionalmente, estos resultados demuestran que en el 7 mo año y la Residencia Médica, el tiempo durante el pase de visita, consulta externa y tiempo en el quirófano, se desarrolla con mayor necesidad para culminar con los deberes clínicos que llevar a cabo la labor docente. La principal causa es la sobrecarga laboral de los médicos docentes/asistenciales y como resultado se pierde la enseñanza al lado del paciente que ha sido considera históricamente fundamental en medicina. ${ }^{13} \mathrm{En}$ estudios realizados en el país se ha demostrado que las horas de trabajo realizadas por los estudiantes son mayores a las dispuestas en sus reglamentos. ${ }^{12}$ Por otra parte, se registró que las sanciones no reglamentadas son un mecanismo utilizado para crear una jerarquía médica, en muchos casos, violentando los derechos humanos y laborales. El acoso laboral y maltrato de manera verbal, física o psicológica provoca daño emocional y no existe acompañamiento psicológico. Las instituciones de educación superior deben diseñar y ejecutar los procesos educativos para transformar el proceso de enseñanza-aprendizaje en un espacio de crecimiento personal, bienestar psicológico y de paz. ${ }^{14}$

Según los resultados los médicos en formacion reciben a diario algún tipo de acoso sexual, de cualquier miembro del establecimiento de salud, incluidos pacientes. Se conoce que el $69 \%$ de las víctimas de acoso pueden presentar un cuadro depresivo severo que amerita tratamiento y presentan riesgo de suicidio. ${ }^{15}$ Actualmente, existen canales diseñados para abordar este tipo de problemáticas, pero no se fomenta una cultura que incentive a la denuncia. El Síndrome de Burnout, es una condición asociada a la depresión y algunos estilos de comportamiento como el consumo de sustancias. ${ }^{16}$ En estudios previos se ha mostrado que en Pediatría el $70 \%$ de los médicos residentes sentía un cansancio emocional alto ${ }^{17}$ y en internado rotatorio se demostró una prevalencia del $87 \%,{ }^{16}$ por lo cual, abordar e intervenir todos los factores que conllevan a los médicos en formación a estados de depresión es de suma importancia ya que existe mayor riesgo de suicidio en la profesión médica.

Sobre la seguridad del paciente es evidente la limitada capacidad para contribuir a reducir la brecha de exclusión en salud ${ }^{18}$ en un sistema de salud colapsado. La sobrecarga laboral ${ }^{12}$ a la que se exponen los médicos en formación y los docentes se traduce en menos horas académicas invertidas, por lo cual, no se adquieren nuevas habilidades teóricoprácticas. Los médicos en formación acuden a los procesos de aprendizaje autodirigido siendo superficial en la mayoría de los casos. ${ }^{10}$ Los médicos en formación se ven forzados a realizar sin supervisión de un médico con experiencia procedimientos que no conocen, esto debido a la sobrecarga laboral y el concepto erróneo de que el médico en formación es indispensable para el funcionamiento de un servicio.

Los cambios de guardias representan un elemento importante en la conducta y aprendizaje ético sobre el principio de confidencialidad, sin embargo, no se llevan a cabo en la mayoría de los establecimientos. Velar por la adecuada transmisión de la información en el cambio de guardia evita la omisión de detalles críticos sobre la situación de un paciente. La comunicación ineficaz ha sido comprobada como la responsable del $25 \%$ a $40 \%$ de los eventos adversos en el cuidado de los pacientes en los servicios de salud. ${ }^{19}$ Además, existe divulgación a través de redes sociales y el seguimiento se limita a una reunión de personal. Tampoco existe los canales de comunicación para denunciar que sean promocionados al paciente durante su experiencia hospitalaria y evitar este tipo de prácticas, y así, evitar la recurrencia. La retroalimentación o la capacidad de que un paciente pueda colocar una queja es de suma importancia para mejorar la seguridad y calidad de atención que reciben los pacientes. 
Los protocolos no están presentes en la mayoría de los centros asistenciales. La importancia de los protocolos o guías clínicas estandarizadas es el potencial para mejorar tanto la calidad o el proceso de cuidado del paciente y los resultados de este..$^{20} \mathrm{La}$ supervisión que contribuye a la retroalimentación para estudiante es de suma importancia en su formación y seguridad del paciente. ${ }^{21}$ El Síndrome de Burnout ha demostrado una relación negativa con la calidad del cuidado del paciente, entre mayor grado de Síndrome de Burnout exista, peor es la calidad del cuidado que recibe el paciente reduciendo la seguridad de este. $^{22}$

La mayoría de los incidentes son prevenibles y se puede evitar su recurrencia al fortalecer la formación médica y canales para las quejas del paciente..$^{23} \mathrm{La}$ OMS define la seguridad del paciente como la ausencia de daños evitables a un paciente y la reducción del riesgo de daños innecesarios durante el proceso de atención médica, ${ }^{24}$ lo antes discutido pone en evidencia la importancia de modificar ciertos aspectos en la formación médica del país.

Una de las limitantes del estudio fue plataforma que permitió realizar un número reducido de encuestas por grupo de interés debido al alto costo de esta, logrando una fracción de la muestra estimada. Por otro lado, otra limitante fue la falta de estudios de esta dimensión en la región. Se recomienda que este tipo de estudio se realice periódicamente como un esfuerzo interinstitucional para evaluar el impacto de cada intervención realizada en conjunto con las autoridades universitarias, gubernamentales y el Colegio Médico de Honduras, para encaminar el sistema de formación médica y sistema de salud nacional a un modelo más sustentable y académico.

En conclusión, la formación médica en Honduras se ve afectada por factores que pueden ser modificados mediante intervenciones y apego a las normativas establecidas. El im- pacto final se traduce en personal en formación en un modelo deteriorado que no logra explotar todo el potencial, que sufre de repercusiones emocionales catastróficas como la depresión ${ }^{25}$ que luego se exacerba debido al alto índice de desempleo al que se enfrentan los médicos al graduarse, además, los pacientes se ven expuestos a daños evitables durante su experiencia hospitalaria. Es importante que ante la formación de cualquier profesional de la salud preguntarnos, ¿a favor de qué (mundo) y de quién (persona) formamos? La Organización Mundial de la Salud (OMS) exhorta a establecer medidas que estén orientadas hacia un desarrollo educativo para una atención equitativa, eficaz e inclusiva a los pacientes, familias y comunidades, en adecuación con las necesidades y valores de cada sociedad. ${ }^{26}$ Se recomienda la introducción del modelo de la pirámide invertida de Cambridge junto con la educación interprofesional en salud en Honduras, para contribuir a mejorar la calidad de la educación en el aspecto humano. ${ }^{27,28}$ Por otro lado, es de vital importancia recuperar el profesionalismo en la educación médica del país, cuya infraestructura consiste en las competencias clínicas, habilidades de comunicación con pacientes y colegas y un verdadero entendimiento de los aspectos éticos y legales de la medicina. La construcción desde esta base compone los atributos del profesionalismo: responsabilidad, altruismo, competencia y con mayor importancia, humanismo. ${ }^{29,30}$

\section{AGRADECIMIENTOS}

Se reconoce y agradece el apoyo brindado por la Junta Directiva del Colegio Médico de Honduras 2018-2020 mediante logística y acompañamiento para llevar a cabo el estudio. Una vez publicado este artículo, la encuesta será de libre acceso para la evaluación periódica de la educación médica por parte del Colegio Médico de Honduras.

\section{REFERENCIAS}

1. General Medical Council (UK). National Training Surveys Reports.2013-2018. [Internet] Reino Unido: GMC; 2019. [Acceso 10 de Septiembre 2019]. Disponible en: https://www.gmc-uk.org/about/what-we-doand-why/data-and-research/national-training-surveys-reports.

2. Wen S, Xu J, Carline JD, Zhong F, Zhong Y, Shen S. Effects of a teaching evaluation system: a case study. Int J Med Educ. 2011; 2:18-2.

3. Urrutia-Aguilar E, Aburto-Arciniega MB, Arce-Cedeño A, GuevaraGuzmán R. Teaching training assessed through mixed-method. FEM [Internet]. 2015 [Acceso 15 de septiembre 2019]; 18(5):337-343. Disponible en: http://scielo.isciii.es/scielo.php?script=sci_arttext\&pid=S201498322015000600008\&lng=en\&tlng=en

4. Avendaño CA, Gutiérrez KA, Salgado CF, Dos-Santos MA. Rendimiento Académico en Estudiantes de Ingeniería Comercial: Modelo por Competencias y Factores de Influencia. Formación universitaria. [Internet].; 2016 [Acceso 31 de agosto 2019];9(3):3-10. Disponible en: https://dx.doi. org/10.4067/S0718-50062016000300002.

5. Reseña Historia de la Facultad de Medicina. Biblioteca Virtual en Salud. [Internet]. Honduras: snt. [Acceso 29 de agosto 2019]. Disponible en: http://www.bvs.hn/Honduras/Historia/ResenaHistoriaFCM.pdf.

6. Espinoza Murra D. Evolución histórica de la Facultad de Ciencias. Rev
Méd Hondur. 2005; 73(supl.2):S37-S44.

7. González-Montero M, Lara-Gallardo P, González Martínez J. Modelos educativos en medicina y su evolución histórica. Rev Esp Méd Quir. 2015; 20(256-265)

8. García-García JA, González-Martínez JF, Estrada-Aguilar L, Uriega-González PS. Educación médica basada en competencias. Rev Méd Hosp Gen Méx. 2010; 73(57-69).

9. Arpita Rojas AM. Síndrome de Burnout mediante la aplicación del cuestionario "Maslach Burnout Inventory" en internos de Obstetricia del Instituto Nacional Materno Perinatal y Hospital Nacional Docente Madre Niño "San Bartolomé". [Tesis en línea] Lima, Perú: Universidad Nacional Mayor de San Marcos; 2016. [Acceso 31 de agosto 2019]. Disponible en: http://cybertesis.unmsm.edu.pe/handle/cybertesis/5441

10. McLean M, Gibbs TJ. Learner-centred medical education: Improved learning or increased stress? Educ Health: Abingdon).2009;22(3):287.

11. Honduras. Secretaría de trabajo y seguridad Social. Código del Trabajo de Honduras. Decreto No. 189 de 1959. Edición rubricada y concordada con las normas nacionales e internacionales del trabajo. Matute López DE, Rodríguez Mejía, editores. [Tegucigalpa:Impresos Ruiz; 2009].p.137-141.

12. Diaz-Valle DJ, Rivas-Sevilla K, Yanez Salguero V, Ramirez-Izcoa A, Valle- 
Reconco JA. Volumen de atenciones, características académicas y económicas de estudiantes internos, en servicio social y residentes. Rev Fac Cienc Méd. 2016; 13(2):(27-36).

13. AlMutar S, AlTourah L, Sadeq H, Karim J, Marwan Y. Medical and surgical ward rounds in teaching hospitals of kuwait university: students' perceptions. Adv Med Educ Pract. 2013; 4:189-193.

14. Sandoval Barrientos S, Dorner París A, Véliz Burgos A. Bienestar psicológico en estudiantes de carreras de la salud. Inv Educ Méd. 2017; 6(24):260-266.

15. Cabrera-Gómez C, Caldas-Luzeiro J, Rivera-Porras D, Carrillo-Sierra $\mathrm{S}$. Diseño del programa de bienestar psicológico de educación en salud online "Florece" para contextos universitarios. Arch Venez Farmacol Ter. 2019;38(5):533-539.

16. Reyes Ticas A, Medina MT, Mesa X, Paredes Y, Barahona Y, Sierra M. Estudio de Síndrome de "Burnout", Depresión y Factores Asociados en los Practicantes Internos del Hospital Escuela. Rev Fac Cienc Méd. 2012; 9(14-20).

17. Bonilla Medina PS. Síndrome de Burnout en estudiantes del Post grado de Pediatría. [Tesis en línea] Cortés, Honduras: Universidad Nacional Autónoma de Honduras en el Valle de Sula;2016. [Acceso 31 de agosto 2019]. Disponible en: http://www.bvs.hn/TMVS/pdf/TMVS27/pdf/TMVS27.pdf

18. Carménate-Milián L, Herrera-Ramos A, Ramos-Cáceres D. Situación del Sistema de Salud en Honduras y el Nuevo Modelo de Salud Propuesto. Arch Med. [Internet]. 2016 [Acceso 31 de agosto 2019]. Disponible en: http://www.archivosdemedicina.com/medicina-de-familia/situacioacutendel-sistema-de-salud-en-honduras-y-el-nuevo-modelo-de-salud-propuesto.php?aid $=17878$

19. Eggins S, Slade D. Communication in Clinical Handover: Improving the Safety and Quality of the Patient Experience. J Public Health Res. 2015; 4(3):666.

20. Graham ID, Harrison MB. Evaluation and adaptation of clinical practice guidelines. Evid Based Nurs. 2005; 8(3): 68-72.

21. Qureshi NS. Giving effective feedback in medical education. Obstetr Gynaecol. 2017; 19(3):243- 8. DOI:10.1111/tog.12391

22. Salyers MP, Bonfils KA, Luther L, Firmin R, White DA, Adams EL, et al. The Relationship Between Professional Burnout and Quality and Safety in Healthcare: A Meta-Analysis. J Gen Intern Med. 2017;32(475).

23. Cave J, Dacre J. Dealing with complaints. BMJ. 2008; 336: 326-328.

24. World Health Organization. Patient safety. [Internet]. Ginebra: WHO; 2019 [Acceso 17 de septiembre 2019]. Disponible en: https://www.who.int/patientsafety/en/

25. Guerrero López JB, Heinze Martin G, Ortiz de León S, Cortés Morelos J, Barragán Pérez V, Flore-Ramos M. Factores que predicen depresión en estudiantes. Gac Méd Méx. 2013; 149:598-604.

26. Morán-Barrios J, Ruiz de Gauna Bahillo P. ¿Reinventar la formación de médicos especialistas?: Principios y retos. Nefrología (Madrid). 2010;30(6):604-612.

27. Organización Panamericana de Salud, Organización Mundial de la Salud, Observatorio Regional de Recurso Humano de Salud. Educación interpersonal. [Internet]. Washington D.C.: OPS/OMS; 2019 [Acceso 17 de septiembre 2019]. Disponible en: https://www.observatoriorh.org/educacioninterprofesional-eip.

28. Organización Panamericana de Salud/ Organización Mundial de la Salud. OPS Honduras. Jornada sobre educación interprofesional en salud en Honduras [Internet]. Washington D.C.: OPS/OMS; 2019 [Acceso 17 de septiembre 2019]. Disponible en: https://www.paho.org/hon/index. php?option=com_content\&view=article\&id=1703:educacion-interprofesional-en-salud-se-socializa-plan-entre-decanos-y-jefes-de\&ltemid=226.

29. Mueller PS. Incorporating Professionalism into Medical Education: The Mayo Clinic Experience. Keio J Med. 2009;58(3):133-143.

30. Zheng S, Shi S. Medical education and medical professionalism in China. The Lancet 2016;387(10025):1272-1273.

ABSTRACT. Background: The health system in Honduras contributes to medical training in an important manner, providing access to the facilities, staff and patients. Objective: Describe the perception of medical trainees and trainers about factors that can affect medical education and patient safety, Honduras, 2019. Methods: Cross-sectional study on medical students (seventh and eighth year), medical residents and trainers from three universities. We estimated a randomized sample. An online survey was conducted through a web platform available for one week. Results: In total there were 324 participants, $205(63.3 \%)$ medical students (seventh and eighth year), $102(31.5 \%)$ medical residents and $17(5.3 \%)$ trainers; $53.4 \%$ (173) were female. $33.9 \%(104)$ informed being sexually harass, $38.8 \%$ (113) receiving unregulated sanctions, $47.9 \%$ (147) performed procedures not taught and without supervision. 58.8\% (10) of trainer considered assigned time to teach insufficient in contrast with other trainer's obligations. $42.3 \%$ (130) of students and $11.8 \%$ (2) of trainers presented with severe Burnout Syndrome. Work overload was associated to not acquiring new skills in 7 th year medical students (OR 2.5, IC95\% 1.2-3.7, $p=0.02$ ), and not attending mandatory teaching in seventh year medical students and medical residents in $75.1 \%$ (OR 3.4, IC95\% 2.82-3.98, $p<0.00001$ ). Discussion: Work overload, sexual and labour harassment, unregulated sanctions and Burnout Syndrome are modifiable factors that can contribute to an urgent improvement of medical education in Honduras.

Keywords: Burnout, psychological; Medical education; Competency-based education; Patient safety. 